\title{
A Social Uncertainty Principle with Application to Principal Agent Problems
}

\author{
Jesse Hoey \\ David R. Cheriton School of Computer Science \\ University of Waterloo
}

March 17, 2022

\begin{abstract}
In this paper, I consider the complementarity of concrete and abstract construals, and show how it relates to problems of group coordination, specifically principal agent problems. I base this argument on evidence that the brain is composed of deeply interlocking layers which alternate in representing things concretely (direct correspondence to sensors), or abstractly (allowing for mental travel). I discuss how this complementarity, when applied to problems of collective behavior, leads to a social uncertainty principle in which a situation may not be modeled arbitrarily precisely both concretely and abstractly. Further, both abstract and concrete construals are intimately tied to action, the ultimate goal of any intelligent agent's neural system. The key message in this paper is that the complementarity between functional levels induces a collective choice of how uncertainty is managed in individual minds, and in enclosing or external groups, and leads to important differences in the cooperative behaviors of individuals and groups. I also describe a computational model of an instance of this process, and discuss the complementarity of principal agent problems.
\end{abstract}

Prefatorial remarks:The ideas presented in this paper are distinct from theories of "quantum cognition" or the "quantum brain," and "quantum" effects are not a part of what I am presenting. I discuss this distinction further in Section C. Further, I am not denying philosophical realism in this paper, only stating that meaning may not be solely approximation to a reality independent of the mind, but rather may also include a description of the interactions of the perceiver. I am proposing a view of reality that is a continuous blend of pragmatism and realism. This comes about by viewing individuals as both objective (individual) and solidary (group-oriented) at the same time.

\section{Introduction}

Consider the five rocks in Figure 1. I'm only talking about the five medium sized rocks in the center of the picture, not all the other smaller and bigger rocks around. They are all rocks, and are therefore all the same, in some way. At the same time, they are not all the same! One is sort of more round-ish and one is sort of more square-ish, for example. The rocks have some variation that I am ignoring by labeling them rocks. Why do I choose this variation and not another? I argue that the selection of this variation is a key element of human cognition, tightly tied to an individual's behavior. I might consider throwing one of the rocks in Figure 1 into the lake, or making a cairn out of them. If I go to see Uluru (the biggest monolithic rock in the world), its meaning as a rock will be very different to me, so taking a picture may be a 


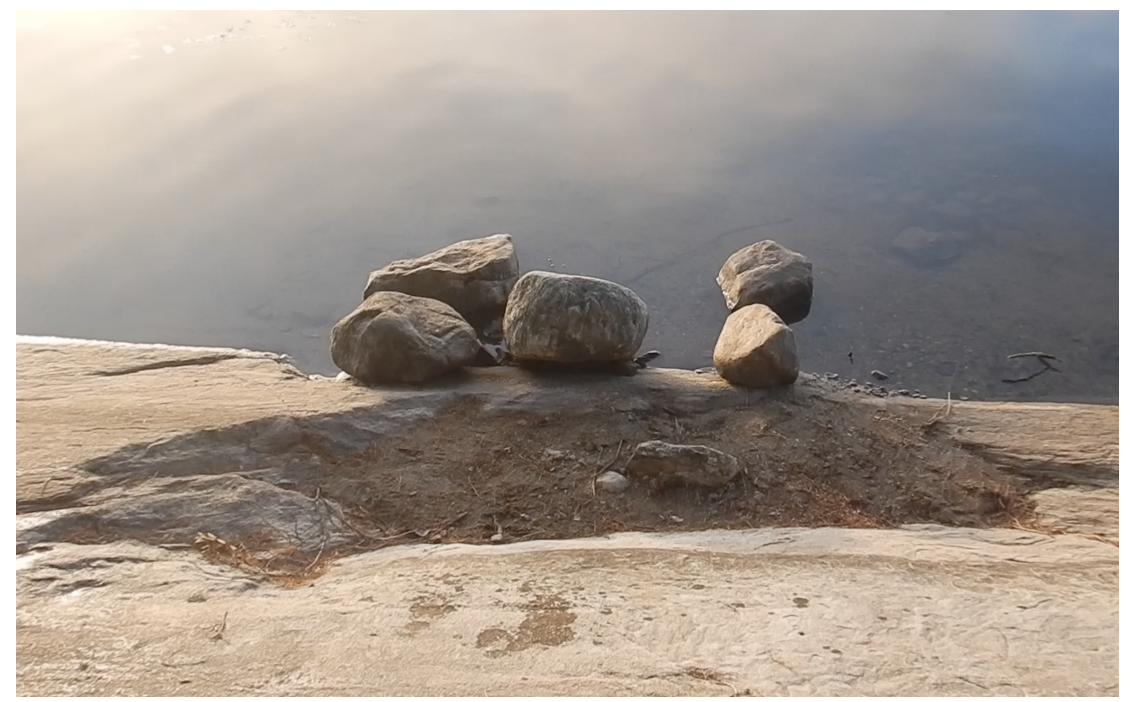

Figure 1: Five Rocks.

more salient action (even though in this case I would have to stop myself from doing so). The meaning would be different again for a person who considers this rock to be sacred, and they might consider performing a ritual.

How do people construe this agent-object relation and why is such meaning even needed? Consider that action, as a sequence of behaviors, is a primary goal of an agent's information processing. To take action, an agent needs sufficient precision in beliefs, or an epistemological certainty sufficient to distinguish the effects of different actions. To achieve this precision, an agent can gather evidence, but may run into a computational or temporal bound (the situation is too complex or happening too fast to be modeled completely), or an information bound. Static rocks on a shore are not all that relevant to me, but consider if they were five hungry polar bears. I would suddenly gain a big advantage by assuming the bears are identical and so my response should be flight. If I tried to deliberatively analyze which one was the hungriest, I would likely not survive. Therefore, I allow my "agent," nature, to hide information from me, acting as the "principal." 1 The information that is hidden is the less important stuff to me. The fact that the polar bear who is attacking me has some mud stuck to its fur does not matter to me at this time. This information bound is even more complicated in social situations, where information may be hidden deliberately $\left.\right|^{2}$

Therefore, the abstract concept of a "rock" is related directly to the change in the perceiver's actions with respect to the set of all rocks. It represents how much effort is needed to map from actual, literal evidence, to the perceiver's behavior: I can tell the difference between a small rock and a big rock because I can lift one and not the other. This is an instance of a more general principle which says the meaning of an entity is the change in action required for a specific instance of that entity as compared to the action required for the generic instance. The generic instance may be culturally defined. This more general principle is the complementarity

\footnotetext{
${ }^{1}$ Principal and agent refer to the two sides of this information asymmetry. Normally the principal has some economic advantage to gain from the actions of the agent, but we can ignore this for now in order to focus on only the informational asymmetry.

${ }^{2}$ Although this also happens in other animals, e.g. camouflage.
} 
or duality of meaning and being (James, 1890). Consider how humans know both what something is (ontology) and what something means (epistemology). However, if I am more sure of what something is, I am less sure what it means $\mathrm{H}^{3}$ This idea has been extensively tested in practice as the decreased reliance on abstract construals (gestalts, generality, morality, values, theory) with decreased mental distance (Shapira et al., 2012). True empiricists, as a canonical example, would agree that what something means is nonsense in any case, so can be ignored (is maximally variant). On the other hand, if I am less sure what something is, then I'm more sure what it means. The canonical example is a deity, as the meaning of things unknown and unknowable. Divinity does not exist in the objective world, but is infinitely precisely defined subjectively, because any evidence may be construed as fitting a deity's will.

Put another way, once I have sufficient precision to choose an action, I stop caring about where it came from and just try to fill in the details in the other complementary view. If I know precisely what something is 4 then I already know what to do, and have no need for any other interpretation or meaning. On the other hand, if I know precisely what something means, I no longer need any evidence. The apocryphal story about the judge who was asked how to identify pornography demonstrates this well. The judge's reply was "I don't know how to identify pornography, but when I see it, I know it." The judge is sufficiently certain about how something feels to take action (e.g. to censure), and no longer needs to analyze the evidence at all. In hindsight, the judge can look at the evidence and pick that which best fits his interpretation, but this is only for convincing others, not the self (Mercier and Sperber, 2017). Similarly, science is the process of uncovering un-truths, so the fallacious meme "science is truth" is another example of this type of high bias reasoning. Moving beyond rocks to social situations, an information asymmetry arises because each individual can’t know everything about all others. Mental travel, or the ability to think about what is unknown, is used to overcome the barriers of social situations that arise because of this asymmetry, and requires abstraction (Gilead et al., 2020).

To summarize, I aim to show that two key levels of processing in the brain must be coupled by a duality that presents a variable "setting" switch that is coupled across members of a group 5

\footnotetext{
${ }^{3}$ Metaphor and (literal) language also have this duality, in that one can't have both at once. It is not possible to speak both literally and metaphorically at the same time, simply because to speak literally is defined as being the act of using non-metaphorical speech, and to speak metaphorically is defined as being the act of using non-literal speech. However, metaphor is thought to be a key facilitator for mental travel (Holyoak and Thagard, 1996). Similarity and difference are also such a duality. One is similar to one's group, but is also different than the group. Individual behavior while in the group is different than while outside of it (Goffman, 1959). The group's behavior is different based on who is currently in it. Finally, induction and deduction share the same duality. One cannot deduce what one needs to induce, and vice versa. Without a model, there is nothing to deduce. Without evidence, there is nothing to induce. It is only the combination of both that yields something, and this combination can be done in an infinitude of ways. This infinitude is what we label "ideas," or "concepts."

${ }^{4}$ This must include everything about my history of interactions with the object and all surrounding circumstances, a difficult task.

${ }^{5} \mathrm{I}$ do not wade into debates about how symbols are represented in the brain or in a computer. You may implement both forms of processing using a network of spiking neurons and glial cells, or using a colony of live ants, if you wish. That is, "symbol-like" operations are compatible with a connectionist (or any other) approach, if one considers the problem in a less reductionist light. I will be circumventing the symbol grounding problem by conjecturing that what we describe as symbols are not symbols at all but rather socially shared derivatives of the action space of an agent, with language used for communication to other humans. Thus, a symbol is a linguistic construct only, that we use to refer in a communicable way to the reality that is this derivative function. I do not address where or how these functions are stored. Further, my aim is primarily to relate this notion of complementarity to two levels of processing that correspond roughly to language and cultural structure, or to objective construals of the social world and subjective impressions of social interactions. Although some of the
} 
The setting used by an agent, and by a group, are critical to determining the relative orientation of the two sides in any exchange. In the following section, I review the general idea of a duality and conjugate variables, and then in Section 3 and Section 4 delve more deeply into socialpsychological and technical perspectives, respectively. I then describe a specific computational model of this complementarity, and I show how this model can be applied to the principal agent problem.

\section{Complementarity}

In this section, I review the basics of conjugate variables, and then discuss how their complementarity may be represented in the human mind, drawing on (Gilead et al., 2020).

\subsection{Conjugate Variables}

Conjugate variables are sets of variables related in the same way that frequency and time are related by the Fourier transform. Conjugate variables exhibit a complementarity, in that a signal can be represented in one space or the other, but not both or neither. Similarly, when modeling, it is more helpful to view the variables in one or the other depending on the situation. Technically, features 1 and 2 of some object are conjugate if

feature 1 = derivatives of the object's action with respect to feature 2.

That is, if feature 1 is defined as the rate of change of an object's action with respect to the state of object feature 2, then features 1 and 2 exhibit complementarity because, as the measurement precision of one feature increases, the precision of the other must decrease. The definition causes the complementarity: one cannot have a static object that changes, or "you can't step into the same river twice. '7

\subsection{Complementarity in the brain}

Gilead et al. (2020) describe a model of the functioning of the human brain as a hierarchical set of levels of abstraction, each of which has a two-fold structure. The abstractum is a general description of the situation at some level, while the concretum is the specifics of the situation, at many levels in the brain. The concreta in early vision are the signals emitted on the retinal nerve, while the V1 cortical neurons are an abstracta of these signals as a series of edges or corners. Three types of representational abilities of the brain are also discussed: modalityspecific, multi-modal, and categorical. It is the categorical abstractions that form the primary sources for mental travel, or the ability to put oneself in an imaginary situation (e.g. as someone else). However, they may do so in many ways, including analogical transfer and cultural transfer.

concepts could be applied at other levels (say visual processing of gestalts), I leave this for future work.

${ }^{6}$ The action describes how a physical system changes over time, and is significant in physics because according to the principle of least action, the equations of motion for an object are those that describe the trajectory with the least action. The action is the integral of the Lagrangian, which describes the flow of energy. Thus, the change in action is a change in a sum of energy over time. One can think of it as the change in the object over time, but also as the behaviors of the actor in context (which can be mapped to change over time).

${ }^{7}$ Attributed by Plato to Heraclitus in Theaetetus (Russell, 1945). 
Neurophysiologically, the first two representations rely more heavily on the hippocampus and sensory cortices, while the categorical relies on the left-lateral frontotemporal cortex (Gilead et al. 2020, p.16).

A pair of concreta and abstracta lie in two distributed functional networks in the brain, but are coupled by potentially more layers of concreta and abstracta. They are mutually exclusive in the sense that information can be represented in one or the other, but not both. Shapira et al. (2012) review this extensively as an "accuracy/detail" tradeoff that relates to the bias variance tradeoff in machine learning. As something unknown is represented in greater and greater detail (lower bias model, less abstraction), it will be more variant in the world (higher variance), meaning that training the model of different instances of the same abstract category will result in different models. As the unknown is represented more and more abstractly (higher bias model), it will be less variant in the world. Gilead et al. (2020) use an example in which someone is trying to predict what their date will look like. A low bias, high variance model would be a complete description of all the characteristics of the date (e.g. 5'11", blonde hair, blue eyes), but it would often be wrong. A high bias model would be "like a human," which would be a poor description of any specific date, but would be correct in almost all cases (low variance). Thus, the act of abstraction reframes the data and allows it to be handled at the same level of precision, but in the abstracted space. To take a nearly trivial example, imagine a template model of a dataset (a set of samples drawn from a distribution in some space). If the distribution becomes more dispersed (covers more of the space), more samples will likely be needed to represent it. If too many samples are needed, and the system runs into a computational wall, then some abstract version of the distribution (say its mean and variance) is passed to a higher level to help guide the placement of the limited number of samples. Further, Shapira et al. (2012), as in grounded/embodied cognition models (Barsalou, 1999), explicitly point out that categorization depends on what goal is in mind. This reflects earlier theorizing in which "Every meaningful statement may be regarded as determining a correlation between desire and action" (Kaplan, 1964 , p.43). Concepts are construed in part as interaction with the self or others.

Thus, I conjecture that the abstractum and the concretum of Gilead et al. (2020) are conjugate variables. If I swap the definition of conjugate variables with these concepts, I get

$$
\text { abstractum }=\text { derivative of action with respect to concretum. }
$$

That is, the abstractum of a concept with respect to an action is the change of that action with respect to the concrete instances of that concept. In the mind, the action is more broadly defined as change in mental state, emotional state, or physical action. If I intend to throw a rock into the lake, then the default action is the sequence of events that will occur between me and my default throwing rock and lake. The rock in Figure 1 is larger than this default rock, and so when I construe the word "rock" in relation to this particular rock and to the act of throwing, I am conceptualizing the additional effort that it would take me to launch this rock.

A concept like "rock" is often interpreted as an abstract (in an agent's head) category of individual things (in the world) composed of a set of features or properties of the object (e.g. size, shape, roughness, etc) (Binder et al., 2016). In this view, agents understand what a rock means by the relationships between its features and those of other objects (e.g. since the density of a rock is greater than the density of water, the rock will sink in the water, which is what I want, so I should throw the rock). In contrast, what I am suggesting is that what primarily defines rocks 
are the things I can do with them. My abstract concept of "rock," in the presence of a real rock and a real intent on my part (e.g., to throw it in the lake), is then a construal of the action that will ensue given one particular history (mine) upon one particular rock. In fact, the concept of "rock" is all such definitions, and therefore must be bounded in the agent's action space, not in the space of the objective world, due to computational bounds. Another way to say this is that something ceases to be a rock when my functional relationship changes sufficiently to call for a change in my policy of action. I am free to change my functional relationship, should I choose to look at this large flat rock (which is much too large to throw) as something I could eat off of. At this point, I may stop calling it a "rock" and start calling it a "table" if I wanted to explain it to someone else. Regardless, the meaning of its label has changed because I changed my functional relationship to it.

Consider also that neural processing (projection, posterior calculation) of abstracta and concreta will mutually affect each other, but the order in which they occur is significant. Once a meaning has been ascribed to an object, perceptions of that object may be different (Shaffer and Flint, 2011). Conversely, changing perceptions will change the meaning ascribed. If I know exactly what the situation is (to precise detail, so this rules out most interactions with humans), then the abstractum is not necessary, and so is maximally variant. However, if I have no idea what the situation is, the abstractum is necessary and is more precisely defined. Conversely, if the abstractum is perfectly defined, all possible changes in the concretum would be specified, and it would become maximally variant. If the abstractum is loosely defined, we can more precisely specify a single concretum. What this implies is that the degree of variation in abstractum and concretum must be related by a (non-quantum) uncertainty principle:

$$
\Delta A+\Delta C \sim_{\epsilon} \sigma^{2}
$$

where $A=$ abstractum and $C=$ concretum, $\Delta$ denotes the capacity of the model space (variance), $\sigma^{2}$ is the actual variance in the agent's environment. The sum of capacities of the two model spaces must be approximately within $\epsilon$ of $\sigma^{2}$ (see Appendix A), where $\epsilon$ is the range of the agent (how bad can it allow its predictions to be). Thus, decreasing the variance of one of $A$ or $C$ means the variance of the other must increase to be able to handle to variance in the environment. If the variance in the environment changes, then one or both of $\Delta A$ or $\Delta C$ also has to change in the same direction (or else $\sigma^{2}$ must be changed). Equation (2) is known as the bias-variance tradeoff in machine learning. It is possible to learn high bias models that are poor predictors, but do not change much with new data (have low variance), or to learn low bias models that are good predictors but that change a lot with new data (high variance).

If the object in question is a person, things get more complicated, but the same tradeoff applies. For example, I conceptualize my postperson as not far from the default as she consistently delivers my mail. However, should I discover she shares an interest with me, then a host of other factors differentiate her from the average postperson. Since my action has changed (from passively receiving mail to actively pursuing a friendship, say), the concreta at play no longer have much to do with mail, but rather on how I should approach this particular person with a cooperative intent. The abstracta change as well from business arrangement to friendship, making different action sets appear.

Equation (2) may be used to give a simple interpretation to the results in (Shapira et al., 2012), in which it is shown that people use a more abstract categorization in situations of increased uncertainty. Indeed, increases in concrete uncertainty would lead to $\Delta C$ increasing, 
which according to Equation (2) implies that $\Delta A$ must be decreasing. As $\Delta A$ is associated with modeling capacity (complexity) this implies that the abstracta will be forced to use simpler models because the data is reframed by the abstraction process. As uncertainty grows in the concreta, any excess beyond the what this level of concreta can handle will be abstracted using Equation (1), "passed up" to to become the next level's concreta, and so on.

\section{Making it concrete...}

I now describe a computational model based in part on the two-level uncertainty relations that were reviewed in the previous section. Any such model has two levels that are complementary, and involves action or behavior.

Equation (2) says that the abstracta are the action response to a change in concreta. Suppose that the category of things I call "rock" has one attribute: size, which I know is directly proportional to weight, given that density and shape are not attributes. If my intent is to lift the rock, I need to apply a force greater than $g \times w(x)$, where $g$ is gravity and $w($.$) is a "weight"$ mapping from size $(x)$ to weight. Suppose the value of this force depends on some action signal $a$ that I have to send my arm muscles, then to lift the rock I must have $f(a)=g w(x)$, where $f$ is a forcing function that maps my actions $a$ to an actual force applied by my arms. I can therefore establish that 8

$$
\text { "rock" } \propto \frac{d a}{d x}=\frac{d f}{d x} \times \frac{d a}{d f} .
$$

Assuming $\frac{d f}{d x}=$ constant in $a$, how would I measure $\frac{d a}{d f}$ ? By attempting to lift two different sized rocks, with sizes $x_{1}$ and $x_{2}$. I can slowly increase $a$ until $f(a)>g w\left(x_{i}\right)$ for each $i$, record this as $a_{i}$, and write 9

$$
\text { "rock" } \propto \frac{a_{2}-a_{1}}{f\left(a_{2}\right)_{x_{2}}-f\left(a_{1}\right)_{x_{1}}},
$$

where $f(a)_{x}$ means that $f(a)$ is evaluated at $x$ (and so is $g w(x)$ ). Equation (4) implies that meaning vanishes when the action does not change as a function of the state. In turn, this implies that things stop having meaning when they are no longer relevant (when they will not make a difference to the actor's energy budget). The system is at equilibrium, and the energy required is minimal. At this energy minimum, the world conforms to the agent's expectations, and no abstract reasoning is required to take the leap of faith needed in the face of surprise (Friston, 2010).

Things get more complicated if dealing with intelligent agents who can also act. Realist interpretations of meaning see "truth as correspondence to reality" (Rorty, 1991, p.22), such that an object like a "domestic cat" is an animal of a certain size with pointy ears, fur and claws that catches birds and is somewhat of a "loner." Any object found to have these properties will be labeled a "cat." In the interpretation I am presenting, the cat is defined by the different ways that I interact with it, and how these impact my well being. That is, "cat" is the object that I interact with by gently stroking, which puts the cat and I into a mutual relationship of comfort.

\footnotetext{
${ }^{8} \mathrm{I}$ am being slightly loose with the meaning of action $a$ here to simplify the presentation for the purposes of this paper. In fact, the action in this derivative is the integral of energy over time, but assuming a principle of least action, I can equate the two quantities.

${ }^{9} \mathrm{I}$ am writing the derivative as a quotient of macro-level features to ease presentation, but keep in mind that it is actually defined in terms of infinitesimals.
} 
Cats are substitutable for me in this way. However, Tom Sawyer (a very naughty boy) likes to tease cats, and so for him a "cat" is the object that screeches as he torments it.

I remind the reader that the examples of the rocks and cats are deliberately oversimplified. One would likely not need to think of rocks in this way, as they are largely manageable in the concretum. If anything goes wrong, I can simply find another rock or cat. However, when other agents with human-level intelligence are present, the situation changes again. For example, my reaction to someone may change as I discover an interest that is shared, arbitrary and individual. If I average across society, I am likely to find something along the lines of (for persons seeking friends, armed with shared arbitrary interests):

$$
\text { "friend" } \propto \frac{\text { approach }- \text { avoid }}{\text { shared }- \text { not shared }}
$$

which says that the meaning of "friend" is "someone you approach because they share your interests."10 This makes sense because the right side is a counterfactual encoding how I would act differently if the situation were to change. It also is a combination of substitutability and predication. That is, any $Y$ that satisfied the predicate shares_interest $(m e, Y)$ would do. A second complication is that people can manipulate information for individual gain, so the predicate shares_interest may be uncertain. A further example is discussed in Appendix B.

Consider the duality of the individual and group in this light. It is not possible to see oneself as completely individual and completely a group member at the same time: the two are complementary, such that

$$
\text { group } \propto \frac{\text { change in individual action }}{\text { change in group state }} .
$$

This implies that a group is defined by how my action changes as a function of changes to the group. Notice that I, too, am the group, as is everyone else in the group, and therefore the group's definition is not a collection of features of each individual, but rather a motivational process that describes how people should act as group members (Hoey, 2021).

The relative amount of epistemological work done at different levels is a "setting" used by each individual and by the group. How do they all choose the same setting? This is described by an individual being "drawn into" the group - i.e. adjusting their model to match that of the group and vice-versa. As noted by Peirce (1955), even the culturally accepted meanings of things such as measures of weight (i.e. the pound) may be defined by "a compromise [amongst traders] between a desire to retain the old ways and a desire to please new-comers."(Peirce, 1955, p.50). Gilead et al. (2020) point to a desire to achieve a "shared reality" as the motivation for people to overcome social epistemic barriers (e.g. uncertainty over who someone is). In turn, this leads to the emergence of "in-group" and "out-group" sets of people. As noted by Shapira et al. (2012), as situations become more uncertain (as mental distance increases), group membership becomes more salient, pointing to the same complementarity. This sharing process may involve many complexities, including those based on social and network structures (Brush et al., 2013).

\footnotetext{
10 "approach" means the energy required for the entire act of approaching, so a "friend" is defined as how the energy required for behavior changes as a function of the situation.
} 


\section{Philosophical Connections and Practical Solutions}

James (1890) and Peirce (1955), founders of the pragmatic approach, each discussed the nature of meaning as it relates to action. The pragmatic maxim holds that what is represented in the brain, language in particular, is not the objective world outside of us, but rather a complete description of the world outside and inside, and the relationship between the two (Peirce, 1955). My concept of "chair" includes everything that I could sit on, including how I might sit on it. Once I (abstractly) decide something is not a chair, I cross a very rapid gap in my comprehension of the world. This gap is what James' referred to as the "flights and perchings" of thought (James, 1890, p.243), and what Stephenson bases much of his theorizing on (Stephenson, 1986b a). The gaps may also be the basis of transfer learning and mental travel, as I can cross the gap symbolically or metaphorically, and construct potential solutions without having ever encountered the situation. For example, I can imagine what meeting the Queen of England would be like, and decide in advance on a course of action. My expectations might be completely violated, but more flights and perchings would hopefully come to rescue me. Similarly, much phenomenological thinking revolved around the relationship between the world and an embodied agent's perceptual and motor systems (Heidegger, 1927). The Gibsonian view, for example, was direct perception of the affordance of something, or how it is related to the perceiver's actions (Gibson, 1986).

MacKinnon (1994) relates the social psychology of complementarity to Bohr (1950) and James (1890) through Stephenson (1986b a). Stephenson's key idea is to do a factor analysis (called $Q)$ in the space of people (across variables of interest) rather than one (called $R$ ) in the space of variables of interest (across people) (Burt, 1940). In doing so, the complementarity of these two approaches was revealed. In the one, $Q$, the world is viewed in terms of a cultural structure, that is, the inter-relationships between networks of types of people (e.g. a neighbour is someone who is helpful and friendly). In the other, $R$, the world is viewed in terms of a social structure, that is, how specific individuals are connected to each other (e.g. I see my neighbour Chad every day) (Wallace, 1983).

These two different epistemological views of the world are both needed, they are inextricable (MacKinnon, 1994). Further, understanding social and cultural structures requires thinking of people as individuals and groups at the same time. The two are complementary. Thus I may write:

cultural structure $=$ derivative of action with respect to its social structure.

The cultural structure defines how my action should change as a function of changes to the social structure. How my action changes, however, is in fact how everyone's action changes, and so the actions of someone reveals what kind of person they are. That is,

kind of person $Q$ acts more/less a certain way in situation (social structure) $R$.

For example,

A doctor is someone who acts imperatively when interacting with patients in a hospital.

Factoring people in the space of features $(\mathrm{R})$ gives a subspace represented as vectors of features. Factoring features in the space of people $(\mathrm{Q})$ gives a subspace represented as vectors of people.

Consider the size of these spaces. There are potentially thousands of features one can use to 
describe a person: race, age, clothing style, eyelash length, etc. In appraising a person in terms of features, one gets a very high dimensional vector, leading to slow thinking (Kahneman, 2011). In contrast, appraising a person in terms of people, one can use a much lower dimensional space, resulting in fast thinking.

For example, if I see a male doctor in a hospital, I know another doctor (my family doctor), and another older male (my father), and therefore I am able to reason about this current doctor as some kind of combination of my family doctor and my father, and choose an appropriate action based on the way I normally interact with those people (e.g. a deferential one). If instead, I attempt to analyze all the features and pick an action $a=f\left(x_{1}, x_{2}, x_{3}, \ldots, x_{m}\right)$ where $x_{i}$ is the $i^{t h}$ of $m$ features (e.g. age, gender, clothing), more computation will be required, and will generally be slower. Once $a$ is selected using the first method, $f\left(x_{1}, \ldots, x_{m}\right)$ no longer needs to be computed, and instead I only need to justify my chosen action by finding values for at least some (possibly empty) subset of $x_{1}, \ldots, x_{m}$ such that $a=f\left(x_{1}, \ldots, x_{m}\right)$, but I am free to choose $f$. For instance, I might argue that I am being deferential to this doctor because he has gray hair, which means he is old and wise. This type of posthoc reasoning is known to be pervasive (Mercier and Sperber, 2017).

\subsection{Bayesian Affect Control Theory}

Affect control theory (ACT) is a model of emotional coherence based in language (MacKinnon, 1994: Heise, 2007, 2010) that was founded on the control principle of Powers (1973), which states that people try to minimize incongruencies by the deliberate act of controlling their perceptions. Heise transposed this to the sentiment space of Osgood et al. (1957), imposed a structure from symbolic interactionism (Mead, 1934), and added affective dynamics (Gollob, 1974). ACT is a computational model that has been used to predict classes of human behavior in a variety of settings (MacKinnon and Heise, 2010). ACT maintains a deterministic and static denotative model with a semantic modifier-actor-behavior-object-setting structure (e.g. "calm doctor advises angry patient in clinic"), and an associated deterministic but dynamic connotative model: a dynamical system in Osgood's three-dimensional space of affective meaning: evaluation, potency and activity (Osgood et al., 1957). This dynamical system represents values, or evaluative knowledge, which can be contrasted with declarative and procedural knowledge that are represented in the denotative model. The two models (denotative and connotative) are linked with a dictionary that is a mapping from labels (e.g. "doctor") to sentiments (evaluation, potency and activity ratings obtained in large scale population surveys). Actions (behaviors) and identification (labeling of actors) are selected in ACT as those that minimize emotional incongruence.

BayesAct generalizes ACT in three ways (Hoey et al., 2016; Schröder et al., 2016; Hoey et al. 2021; MacKinnon and Hoey, 2021; Hoey, 2021). First, it allows for modeling of variation in sentiment (it is a probabilistic model). Second, it explicitly represents uncertainty over the denotative state (i.e. which label is applied to what situation). Finally, it allows for a preference function, which implements constrained decision theoretic reasoning (Asghar and Hoey, 2015). BayesAct explicitly represents both concrete (denotative) and abstract (connotative), as individual and social factors, in a partially observable Markov decision process (POMDP), implemented using sequential Monte-Carlo sampling. As such, BayesAct is a candidate for implementing a duality of abstracta and concreta at the level of language and sentiment. 
BayesAct combines abstract and concrete at the level of language and sentiment by using a continuous dynamical system as a high bias, low variance model (the connotative process which abstracts social dynamics into simple categories), and then using a low bias, high variance model (the denotative process) within the connotative model space. The key to making this work is that the connotative model is culturally shared, and conformity with it gives security (low variance), but lacks innovative capacity and the ability to handle the unforeseen (high bias). Less conformity with the group lowers the bias, leading to more rational, individualistic thought and more innovation (higher variance). BayesAct obviates the need for artificial "altruism" terms in the preference function of each agent, as is commonly assumed (Fehr and Schmidt, 1999), because each BayesAct agent is an individual optimizer, but only within the space of futures as defined by the cultural structure. In a conflict, for example, two enemies would be culturally (connotatively) expected to fight each other. How such a fight plays out is then handled denotatively including tradeoffs between time and outcomes, and signals of power/dominance (Brush et al., 2018).

BayesAct uses a Bayesian sequential decision making framework and so can implement pure decision-theoretic (rational) reasoning, and non-rational, affective thinking, and anything in between. Further, the POMDP is a substrate for model-based reinforcement learning, and so can be used to simulate future outcomes as in a predictive processing framework (it is a generative model) (Friston, 2010, Hohwy, 2013, Gilead et al., 2020). The free energy principle states something very reminiscent of Powers (1973): cognition and perception are tuned to reduce surprise. Further, the associated active inference approach uses a variational optimization of the expected free energy, and the actions are chosen according to the principle of least action formulated as least surprise. The free energy minimum is the optimal path such that expectations are realized as much as possible.

\subsection{Application to Management}

Given that the world can be described abstractly or concretely, or anything in between, but not as both or as neither, this tradeoff can be observed in groups, and management practices can be adapted to be at least as conducive to matching people than they are to matching skills. An understanding of how uncertainty is handled by each individual and by the group is an important aspect of what behaviors can be expected from the group, including what can be expected to be hidden. A principal needs to know how its messages to its agents are interpreted, and how to interpret the messages coming back. The dualities discussed in this paper can give insights into this problem.

A foundational problem in economics is the principal agent problem (Mansfield and Yohe, 2004) in which lower-level agents in an organizational hierarchy have incentive to hide or alter information sent to higher levels (and vice-versa). The subsequent increase in uncertainty for the principal/owner usually causes a reaction of further symbolic structure imposed from above. The principal's primary aim then is to ensure that agents under their patronage "follow the rules," and the agents usually react by continuing what they were doing, ignoring the rules, and changing what they report to the principal instead. That is, principal agent problems are not solved with modifications to utility functions alone (Fukuyama, 2014). Cooperation is only achievable through the construction of esteem, which involves team, identity or nation building. Identities can be built constructively, by making them attractive in terms of social status or power, or destructively, by, e.g., excluding persons on the basis of physically observable characteristics. 
How can the principal (owner of capital, say) ensure agents are following rules and reporting honestly? There are two ways. She can change the agent's utility function by modifying their pay structure so it rewards or punishes them for rule following, or she can instill the new symbolic structures into the agents through, for example, the development of exercises that build positive team identification (Lawler et al., 2009). This leads to increased abstraction for the agents, allowing them to be more flexible, cooperative and innovative in the concretum. In fact, both may be necessary in order to achieve a cooperative equilibrium. For example, Bury et al. (2019) find that effective climate change mitigation strategies evolve from cash incentives (tax breaks) to motivational messaging (media coverage and public fora devoted to climate change) based on the level of an individual's adoption of mitigation strategies.

The relative management of uncertainty, as well as identities of group members and their emotional understanding of the group situation can be measured using survey methods (as they are in $\mathrm{ACT}$ ), although methods are being developed extract these from document corpora using word embeddings (Loon and Freese, 2021, Kozlowski et al., 2019). This leads to a better understanding on the part of management of both how a team is interpreting the objectives given to them, and to how to better interpret the results coming back. It also calls for diversity across and within teams, to ensure that a breadth of views is incorporated. In sum, the dualities I have exposed in this paper may be used to help solve principal agent problems, by allowing principals to better understand what information is being "left behind" by any of its agents.

\section{Discussion and Conclusion}

There are two parts to the argument I am presenting in this paper. The first is that abstract and concrete processes in the brain are complementary, and so yield an uncertainty relation that intelligent agents must handle. That is, any event may be processed deliberatively (as a set of logical propositions ontologically grounded in the world), or affectively (as an abstract "feeling" of discomfort when confronted with the event holistically), but not both or neither. The abstractions must nevertheless be collective, otherwise they are ineffectual. It is not possible to simultaneously and precisely perceive the forest and the individual trees, yet the trees must be perceived to see the forest. One can describe this with a simple line with a negative slope, as shown in Figure 2. A group (even a society) can organize itself anywhere along this line (e.g. $C o$ and $A n$ in Figure 2) and achieve the same degree of cooperation. However, a group is seldom all in the same place on the line, and, worse, may be split into two or more sub-groups at different places. It is often differences in the specifics of the situation that is confronting subgroups which leads to these splits. Some sub-group facing extreme uncertainty may resort to stronger community ties, for example. In fact, there is a third dimension, one that manages the uncertainty in the connection between deliberative and affective modes, and information being processed by one automatically flows to the other. Once this third dimension is considered, the line in Figure 2 becomes a simplex and there are three mutually intersecting complementarities. Further details may be found in (Hoey, 2021).

The second part of my argument is that the complementarity of abstract and concrete gives us a mechanism to measure their relative uncertainties and to correct for any imbalances, and this mechanism can be instantiated as a POMDP. For example, suppose some team is hiding information from their owner. A remedy may be retributive or restitutive sanctions for rule 


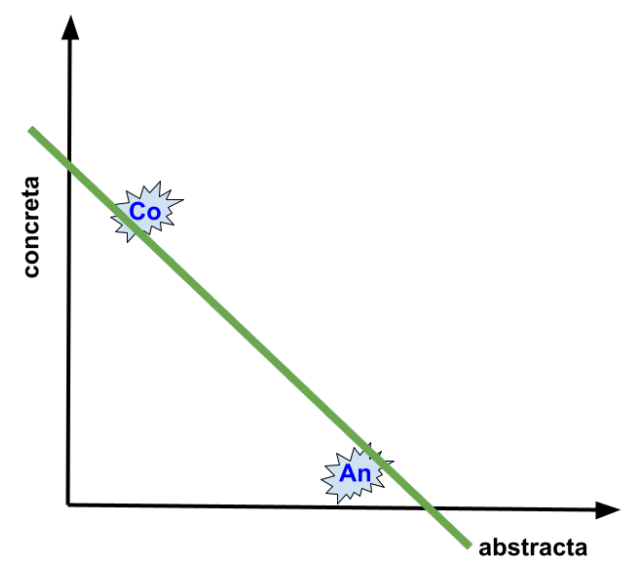

Figure 2: Concrete/abstract tradeoff showing different group locations, Co agents who follow the same strict rules, and $A n$ agents who are free, but must mutually share the same abstractions.

breaking, or positive incentives (e.g. cash rewards) for rule following. However, this does not solve the problem, because although it forces the agents to report things according to a more precise set of rules, it does not force these agents to actually do so. Therefore, it increases uncertainty in the agents without reducing the information asymmetry, and thereby making this coupled pair of levels inefficient. For this dysfunctional team, adding more rules pushes this team to represent additional uncertainty as they have to now embrace two realities: one according to rules, one not. However, their original motivation to free ride on the team remains the same, as the team does not hold much sway in their decision making process.

If, instead, the owner uses positive emotional signals, in which she instills sufficient team spirit in her agents by organizing activities with rewards that generate immediate positive emotional signals (e.g. food, beverage, and leisure). However, she must ensure these emotional rewards are attributed to the team, and so she must first establish a symbolic-like structure such that the agents form an institution or community of practice of their own free will, using their own social capital, which closely aligns with the owner's utility function. Such an effort must involve shared goals and positive rewards (Lawler et al., 2009), along with coordination of the different ways the group members (and all subgroups) are managing uncertainty. A diverse group with a range of such uncertainty management methods is potentially more innovative (Page, 2007), but also requires more effort for coordination (Bales, 1999).

The BayesAct model, as it implements the complementarities I have been discussing at the level of language and sentiment, could be used to either generate simulations or proposals for these symbolic-like structures and emotional signals. This is not a trivial process since the establishment of a community of practice must proceed from the grassroots, from the group members themselves. However, by modeling different individual members and sub-groups, alternate framings of a situation can be automatically evaluated by the model as to their effectiveness. I believe the understanding of how a person and a group manage uncertainty and ambiguity will give important insights into this process. Lastly, simulations with BayesAct can be compared to real data, giving ample falsification avenues. Manually specifying a BayesAct model and checking its simulation outputs against real situations, as is done in (Hoey and Schröder, 2022, Heise, 2013), is one way to seek confirmatory and falsifying evidence. Another is to learn the BayesAct model from one dataset and apply it to another. 
Acknowledgements: This research was support by a Discovery Grant from the Natural Sciences and Engineering Research Council of Canada. I thank Neil MacKinnon, Tobias Schröder and John Levi Martin for helpful comments on early drafts.

\section{References}

Asghar, N. and Hoey, J. (2015). Monte-Carlo planning for socially aligned agents using Bayesian affect control theory. In Proc. Uncertainty in Artificial Intelligence (UAI), pages 72-81. UAI.

Bales, R. F. (1999). Social Interaction Systems: Theory and Measurement. Transaction Publishers, New Brunswick, NJ.

Barsalou, L. (1999). Perceptual symbol systems. Behavioral and Brain Sciences, 22(4):577-660.

Binder, J. R., Conant, L. L., Humphries, C. J., Fernandino, L., Simons, S. B., Aguilar, M., and Desai, R. H. (2016). Toward a brain-based componential semantic representation. Cognitive Neuropsychology, 33(3-4):130-174.

Bohr, N. (1950). On the notions of causality and complementarity. Science, 111:51-54.

Brush, E. R., Krakauer, D. C., and Flack, J. C. (2013). A family of algorithms for computing consensus about node state from network data. PLOS Computational Biology, 9:1-17.

Brush, E. R., Krakauer, D. C., and Flack, J. C. (2018). Conflicts of interest improve collective computation of adaptive social structures. Science Advances, 4(1):e1603311.

Burt, C. L. (1940). The Factors of the Mind: An Introduction to Factor Analysis in Psychology. University of London Press, London.

Bury, T. M., Bauch, C. T., and Anand, M. (2019). Charting pathways to climate change mitigation in a coupled socio-climate model. PLOS Computational Biology, 15(6):1-16.

Busemeyer, J. R. and Pothos, E. M. (2012). Social projection and a quantum approach for behavior in prisoner's dilemma. Psychological Inquiry, 23(1):28-34.

Danilov, V. I. and Lambert-Mogiliansky, A. (2010). Expected utility theory under non-classical uncertainty. Theory and Decision, 68(1):25-47.

Dawes, R. L. (1994). Quantum neurodynamics. IFAC Proceedings Volumes, 27(1):491-495. IFAC Symposium on Modelling and Control in Biomedical Systems, Galveston, TX, USA, 27-30 March 1994.

Dennett, D. (1987). The Intentional Stance. MIT Press, Cambridge, MA.

Denolf, J., Martínez-Martínez, I., Josephy, H., and Barque-Duran, A. (2017). A quantumlike model for complementarity of preferences and beliefs in dilemma games. Journal of Mathematical Psychology, 78:96-106.

Deutsch, D. (1999). Quantum theory of probability and decisions. Proc. R. Soc. Lond. A, 455:3129-3137. 
Doshi, P. and Gmytrasiewicz, P. (2009). Monte-Carlo sampling methods for approximating interactive POMDPs. Journal of Artificial Intelligence Research, 34:297-337.

Fehr, E. and Schmidt, K. M. (1999). A theory of fairness, competition, and cooperation. The Quarterly Journal of Economics, 114(3):817-868.

Friston, K. (2010). The free-energy principle: A unified brain theory? Nature Reviews Neuroscience, 11((2)):127-138.

Fukuyama, F. (2014). Political Order and Political Decay. Farrar, Strauss and Giroux.

Getoor, L. and Taskar, B. (2007). Statistical Relational Learning. MIT Press.

Gibson, J. J. (1986). The Ecological Approach to Visual Perception. Taylor and Francis.

Gigerenzer, G. and Gray, W. D. (2017). A simple heuristic successfully used by humans, animals, and machines: The story of the raf and luftwaffe, hawks and ducks, dogs and frisbees, baseball outfielders and sidewinder missiles - oh my! Topics in Cognitive Science, 9(2):260-263.

Gilead, M., Trope, Y., and Liberman, N. (2020). Above and beyond the concrete: The diverse representational substrates of the predictive brain. Behavioral and Brain Sciences, 43(e121):1-74.

Goffman, E. (1959). The Presentation of Self in Everyday Life. Anchor Books, USA.

Gollob, H. F. (1974). The subject-verb-object approach to social cognition. Psychological Review, $81: 286-321$.

Harnad, S. (1990). The symbol grounding problem. Physica D, 42:335-346.

Heidegger, M. (1927). Being and Time. various.

Heise, D. R. (2007). Expressive Order: Confirming Sentiments in Social Actions. Springer.

Heise, D. R. (2010). Surveying Cultures: Discovering Shared Conceptions and Sentiments. Wiley.

Heise, D. R. (2013). Modeling interactions in small groups. Social Psychology Quarterly, 76:5272 .

Hoey, J. (2021). Equality and freedom as uncertainty in groups. Entropy, 23(11):1384.

Hoey, J., MacKinnon, N., and Schröder, T. (2021). Denotative and connotative control of uncertainty: A computational dual-process model. Judgment and Decision Making, 16(2):505550 .

Hoey, J. and Schröder, T. (2022). Disruption of status orders in societal transitions as affective control of uncertainty. in press, American Behavioral Scientist.

Hoey, J., Schröder, T., and Alhothali, A. (2016). Affect control processes: Intelligent affective interaction using a partially observable Markov decision process. Artificial Intelligence, 230:134-172.

Hohwy, J. (2013). The Predictive Mind. Oxford University Press. 
Holyoak, K. J. and Thagard, P. (1996). Mental Leaps: Analogy in Creative Thought. Garnett.

James, W. (1890). Principles of Psychology. Holt, New York.

Jeffery, K., Pollack, R., and Rovelli, C. (2019). On the statistical mechanics of life: Schrödinger revisited. Entropy, 21(12):1211.

Kahneman, D. (2011). Thinking, Fast and Slow. Doubleday.

Kaplan, A. (1964). The Conduct of Inquiry. Chandler, San Francisco.

Kiverstein, J. (2011). Social understanding without mentalizing. Philosophical Topics, 39(1).

Kozlowski, A. C., Taddy, M., and Evans, J. A. (2019). The geometry of culture: Analyzing the meanings of class through word embeddings. American Sociological Review, 84(5):905-949.

Lawler, E. J., Thye, S. R., and Yoon, J. (2009). Social Commitments in a Depersonalized World. Russell Sage Foundation.

Litt, A., Eliasmith, C., Kroon, F. W., Weinstein, S., and Thagard, P. (2006). Is the brain a quantum computer? Cognitive Science, 30(3):593-603.

Loon, A. V. and Freese, J. (2021). Word embeddings reveal how fundamental sentiments structure natural language. American Behavioral Scientist, In Press.

MacKinnon, N. J. (1994). Symbolic Interactionism as Affect Control. State University of New York Press, Albany.

MacKinnon, N. J. and Heise, D. R. (2010). Self, identity and social institutions. Palgrave and Macmillan, New York, NY.

MacKinnon, N. J. and Hoey, J. (2021). Operationalizing the relation between affect and cognition with the somatic transform. Emotion Review, 13(3):245-256.

Mansfield, E. and Yohe, G. (2004). Microeconomics. Norton, 11 edition.

Mead, G. H. (1934). Mind, Self and Society. University of Chicago Press.

Mercier, H. and Sperber (2017). The Enigma of Reason. Harvard University Press.

Neal, B., Mittal, S., Baratin, A., Tantia, V., Scicluna, M., Lacoste-Julien, S., and Mitliagkas, I. (2018). A modern take on the bias-variance tradeoff in neural networks. CoRR, abs/1810.08591.

Osgood, C. E., Suci, G. J., and Tannenbaum, P. H. (1957). The Measurement of Meaning. University of Illinois Press, Urbana.

Page, S. E. (2007). The Difference: How the power of diversity creates better groups, firms, schools and societies. Princeton University Press.

Peirce, C. (1955). Philosophical Writings of Peirce. Dover Publications, Inc., New York. Selected and edited by Justus Buchler. 
Pothos, E. M. and Busemeyer, J. R. (2022). Quantum cognition. Annual Review of Psychology, $73(1): 749-778$.

Powers, W. T. (1973). Behavior: The control of perception. Aldine publishing co., Chicago.

Rorty, R. (1991). Objectivity, Relativism, and Truth. Cambridge University Press.

Russell, B. (1945). A History of Western Philosophy. Simon and Schuster, Inc.

Schröder, T., Hoey, J., and Rogers, K. B. (2016). Modeling dynamic identities and uncertainty in social interactions: Bayesian affect control theory. American Sociological Review, 81(4):828855 .

Shaffer, D. M. and Flint, M. (2011). Escalating slant: Increasing physiological potential does not reduce slant overestimates. Psychological Science, 22(2):209-211.

Shank, D. B. and Lulham, R. (2016). Products as affective modifiers of identities. Sociological Perspectives.

Shapira, O., ad Yaacov Trope, N. L., and Rim, S. (2012). Levels of mental construal. In Sage Handbook of Social Cognition, pages 229-250. Sage.

Silver, D. and Veness, J. (2010). Monte-carlo planning in large POMDPs. In Lafferty, J., Williams, C., Shawe-Taylor, J., Zemel, R., and Culotta, A., editors, Advances in Neural Information Processing Systems (NeurIPS) 23, pages 2164-2172. Curran Associates, Inc.

Slors, M. (2012). The model-model of the theory-theory. Inquiry, 55(5):521-542.

Stephenson, W. (1986a). William James, Niels Bohr, and complementarity: Ii-pragmatics of a thought. The Psychological Record, 36:529-543.

Stephenson, W. (1986b). William James, Niels Bohr, and complementarity: I-concepts. The Psychological Record, 36:519-527.

Taleb, N. (2007). The Black Swan. Random House.

Vijayakumar, S. (2007). The bias-variance tradeoff. University of Edinburgh. Retrieved 18 February 2022.

Wallace, W. L. (1983). Principles of Scientific Sociology. Aldine Publishing, New York.

\section{A Derivation of the BayesAct social uncertainty principle}

One can fairly easily derive Equation (2) for a basic machine learning of a stochastic function $y=f(x)+\varepsilon$ where $\varepsilon$ is a zero-mean noise process with variance $\sigma^{2}$ and $f(x)$ is a deterministic data generating process(Vijayakumar, 2007). If the estimated function $\hat{f}$ must match the true function, then

$$
\begin{aligned}
E\left[(y-\hat{f})^{2}\right] & =E\left[(f+\varepsilon-\hat{f})^{2}\right] \\
& =(f-E[\hat{f}])^{2}+E\left[(E(\hat{f})-\hat{f})^{2}\right]+\sigma^{2}
\end{aligned}
$$


where I have used the fact that $E(\varepsilon)=0$ and the $E(f)=f$ since $f$ is deterministic. The first term is the expected distance between the models, while the second is the variance in that distance. Thus, the first can be interpreted as the model's bias, Bias $\left([\hat{f}]^{2}\right)$ while the second as the model's variance, $\operatorname{Var}([\hat{f}])$. At its minimum, the expected error $E\left[(y-\hat{f})^{2}\right]$ must be at least $\sigma^{2}$ to be able to properly represent the environment (including unforeseen events), but must not be too big such that everything is missed. Thus, we can extract the uncertainty relation equivalent to Equation (2),

$$
\operatorname{Bias}(\hat{f})+\operatorname{Var}(\hat{f}) \sim_{\epsilon} \sigma^{2},
$$

where I have used the symbol $\sim_{\epsilon}$ to denote that the left side must be approximately within $\epsilon$ of $\sigma^{2}$. The reason this is approximate and deserves a special symbol is that the comparison is made in a value space. That is, the error expectation translates, possibly not smoothly, into a value function which could denote the risk or regret. As the error grows, the distance it can move beyond $\sigma^{2}$ without causing harm to the agent is roughly $\epsilon$, but this depends on the specifics of the problem under consideration. In the BayesAct model, the Bias $(\hat{f})$ term is the denotative model capacity and is denoted by $\delta$, while $\operatorname{Var}(\hat{f})$ is the connotative model capacity, denoted by $\alpha$. As reviewed by Hoey (2021), $\alpha$ and $\delta$ are traded off against each other as a function of the social milieu. Further, a third parameter $\gamma$ is the variance in the transform function between the two levels, and so the bias-variance tradeoffs become a simplex in a three dimensional space.

\section{B Team Sports}

Consider the example of a sports team. A downwards reductionist (realist) view is that the team is defined as

- Player A has skills $A_{1}, A_{2} \ldots$,

- Player B has skills $B_{1}, B_{2}, \ldots$,

- Referee calls penalty $P$ with probability $P_{p} \ldots$

- Game rules are ...

Given all these features, a complete simulation of the game could be obtained, such as one gets in so-called "fantasy" sports video game leagues where all the players are artificial agents. This seems reasonable until you realize that the simulation itself is what is motivating the (robotic) players to act in the video game. Thus, something outside the entire system is involved, invalidating the simulation as insightful to human practice.

In the view I am proposing, the team is defined by how each individual member's actions change as a function of the group's behavior (including the other team, referees, the crowd and the timekeeper). For example, if the superstar player shows up, everyone tries a little harder. The important thing is that the sports team is defined holistically as the sum total of all these changes for all team members, relative to what all others are doing. Motivation is provided by the connotative system which guides action selection and is inherently representative of the group. 


\section{Connection to Other Disciplines}

\section{C.1 Behavioral Economics}

Many approaches to the principal agent problem in economics, game theory and artificial intelligence suggest using a two-factor reward function, with one piece for the individual, and a separate piece, weighted somehow, for others in the group (Fehr and Schmidt, 1999) 11 For example, the average reward function of the group may be supplied to each individual member, and each individual somehow has access to the true, honest preferences of each other agent. However, as we have seen, any relationship between an individual and a group is going to involve some information hiding by the group, since there is only so much each agent can handle. The arguments in support of this artificial assumption usually circumvent this problem with the "a bad map is better than no map" argument, an adage well-known to be patently wrong, as it creates a narrative fallacy that is difficult for people to overcome (Taleb, 2007). This fallacy is often presented as a Hobson's choice: since this is the only map we have, we'll make it look like we chose it on purpose.

In the view I am proposing, complementarities between abstract, culturally based reasoning and concrete reasoning based in the objective world are the main driver of cooperative behavior in groups. The reason these complementarities exist is because of the information asymmetry created by the second law of thermodynamics. As the universe expands, disorder increases and entropy rises, and so information is lost. However, organisms can trap some of this leaking information and create lasting structure from it (Jeffery et al., 2019). A battle for the leaking information amongst primates caused social and cultural structures to emerge and endure due to the evolutionary pressures driven by the increased efficiencies of the more cooperative group. The complementarity then emerges because one can handle this information asymmetry in different ways, which creates social and cultural structures that are highly static and brittle and ones that are highly innovative and flexible. I hinted at this complementarity being behind the ways groups self-organize, and this may be extended to society as a whole, as I have detailed in (Hoey, 2021)

\section{C.2 Quantum Cognition}

Other connections to physics are a bit more opaque. The work of Peirce, James, and Bohr (and others) spawned a research community which attempted to model the brain as a quantum mechanical field (Dawes, 1994), but most now consider this as some form of quantum mysticism, the utility of which is debatable (Litt et al., 2006). More modern treatments of the same idea can be placed under the general umbrella of quantum cognitive theory (Deutsch, 1999, Pothos and Busemeyer, 2022). In such models the complementarity of variables is treated using quantum mechanical operators, but the message remains the same: in a system with complementarities, "quantum" like effects will be observable where things seem to change based on, e.g. the ordering of measurement operators. Here, I show how these may simply be a complementarity tradeoff between between abstract and concrete construals. Denolf et al. (2017) use a sequential game to show this non-commutativity, but I believe the same events could be modeled as simply a change of social situation, also hinted at in the model proposed by (Busemeyer and Pothos,

\footnotetext{
${ }^{11}$ See Hoey et al. (2021) for further discussion of this point.
} 
2012). It is these quanta that are explored in relation to decision theory by Danilov and LambertMogiliansky (2010). The use of non-classical uncertainties (in which there are discontinuities, for example), leads to different decision-making strategies. Here I am proposing an interpretation of this result as being a trade-off between deliberative and affective comprehensions of the state, and relating it to theorizing in social psychology.

The models I am proposing may be treatable using the techniques of quantum cognition, although the need for anything more than uncertainty management is unclear (Litt et al., 2006). While complementarity of denotative and connotative processing in the brain, and the resulting social uncertainty principle that I am presenting have similar properties to the complementarity between physical variables (e.g. position and momentum) and to the Heisenberg uncertainty principle, the connection is merely analogical, as any system with complementary variables at any scale will exhibit the same properties. At the macroscopic (in space and time) level of the brain, actual quantum effects at the microscopic scale are not likely to be very relevant (Litt et al. 2006).

The "quantum brain" hypothesis has a mystical element to it that many scientists fine unpalatable. It uses the analogy of complexity to explain itself: the brain solves hard problems fast, quantum computers solve hard problems fast, so the brain must be a quantum computer. However, there is no need to invoke quantum effects to explain the brain's solution of hard problems. The brain is good at solving hard problems, but not of the same kind as quantum computers at all. While factoring large numbers is not something a human can do any faster than a classical computer, cooperation on international trade agreements and public policy is. The model I am proposing solves the paradox of humans solving these "hard" problems by noting that the cultural structures at play in human negotiation within a certain institution give a rough "oracle"

for behavior in that particular institution. Operating within the guidelines of this oracle allows humans to appear to be solving these hard coordination problems, but in reality they are already solved by the sharing and constant re-definition of cultural structures.

\section{C.3 Explainable AI}

So-called "explainable AI" is often presented as simply identifying the parts of an object or decision as "explaining" the object or decision, allowing for "zero-shot" learning. For example, knowing how to identify a horse, and knowing how to identify stripes, allows one to identify zebras prior to ever having seen one. However, this view is difficult to maintain because the explanation still does not include zebras, only horses with stripes. If I am primarily interested in using the striped horse for transportation, then under this method of identification I would approach the striped horse with an intention to ride it, which likely will not end well. According to Equation (4), the meaning of the striped animal I see is defined by my interaction with it. Thus, my definition of "horse" will either need to change to include these striped, unrideable horses, or I will have to create a "new animal" concept which I will likely stay away from given my uncertainty about how this animal will react to me. In order to talk about my new concept with others, I may invent a word for it and start sharing it with others. 


\section{C.4 Symbol Grounding}

The symbol grounding problem has long stymied psychologists and neuroscientists (Harnad, 1990). Recent attempts to overcome it have led to work on semantic grounding of concepts in brain areas (Binder et al., 2016). That is, there is a correlation between (functional) brain areas and categories of things in the world. In this componential view, objects are recognized because of their parts and relationships to other objects, which may be internal (e.g. emotions). Vehicles and animals are related because they share certain conceptual features (such as movement), and these conceptual features may be combined with other elements to find action responses (e.g. use as transportation). However, although argued to be different, this is still replacing one set of poorly defined constructs with another. The argument by Binder et al. (2016) is that since they can relate the components to functionally separable areas of the brain, then this somehow grounds these components. My view is quite different in that what vehicles and animals share is their ability to be used as a transportation mechanism. Thus, if I am seeking something for companionship, my horse and my dog may become very similar concepts, but if I am looking to go somewhere, my horse might seem a lot more similar to my car than to my dog. Similarly, if I am seeking to play some music, a piano will holistically fit my current explanation, and I will not stop and consider whether the keys are actually connected to the hammers or not before I start playing. Any explanations for how this all happened are left for any required explanation of my behavior to others. Similarly, a house-boat and a house will be considered similar if I plan to live somewhere, but not if I plan to go somewhere. The difference between the concepts house-boat and house is that one affords a nomadic lifestyle, while the other does not. The similarity/difference is swapped if considering a house-boat and a boat (similar for going somewhere, dissimilar for living in). House-boats and boat houses have no similarity.

Another way of seeing the error of the componential approach is that it requires some "weights" for different features in different contexts, but "the mechanism by which such weight adjustments occur is unclear" (Binder et al., 2016, p.33). It is unclear because it is not necessary. In my view, this mechanism is the definition of an abstract concept related to its concrete realization. Thus, situational context effects are not special combinations of features, as claimed by Binder et al. (2016, p.38). In fact, the combinations of features are only post-hoc explanations for why the situational context effects have defined the objects in question through the action space of the agent. To return to zebras, Binder et al. (2016, p.31) state that

"Although barber poles and zebras both have salient visual patterns, they strongly differ in salience on many other attributes (shape complexity, biological motion, body parts, and motion through space) that would preclude them from being considered semantically similar."

I can demonstrate that these differences do not preclude semantic similarity. Barber poles originated in the middle-ages as poles to grip onto when the barber was doing a surgery (which they did in those days). They were often streaked in blood, and so came to symbolize the grisly duties of the barber. Now imagine you lived in this middle-ages world, but that some barbers used live zebras because a serum could be extracted from a live zebra which would dull pain. Imagine further that this method was known to be much more effective than the pole, but was much more expensive (the barber had to maintain and keep the zebra). In this imaginary world, there are two kinds of barbers: those with zebras and those without. Now imagine that, as with

the pole, the zebra tradition had continued but real zebras were replaced with fake ones that 
jutted out above the door of a barber's shop. In this imaginary modern world that derived from our imaginary middle-ages world, barber shops come in two varieties, advertised using either painted zebra statues or painted poles, both of which offer the same services (and neither of which offer any surgeries anymore). However, because of their illustrious past, the "zebra" barbers belong to a special college of licensed barbers who are trained in a special school, while the "pole" barbers are just any enterprising individual who wants to make a living by cutting hair ${ }^{12}$ Now, ask yourself, in this completely believable modern world, could zebras and barber poles, neither of which has changed in shape, size, color, or anything else, be considered semantically similar?

The word "zebra" is not, therefore, simply a combination of "horse" + "stripes" because that ignores the fact that zebras are not rideable. While early treatment of symbol-grounding problems were based on ignoring action and basing all meaning on sets of iconic features and their inter-relationships (Harnad, 1990), the active inference approach sidesteps the issue by grounding in an agent's action space (which is internal), not in the state space (which is external). Thus, "zebra" is revealed as the horse-like object with stripes that is not rideable. Note that the "horse" + "stripes" interpretation of "zebra" is revealed to leave out a second, important, feature that distinguishes zebras from striped horses: what I can do with each of them. What is most interesting about this viewpoint is that the change of meaning bewteen horse and zebra is based on the social structure of the horse, compared to the zebra. While both have a "fight or flight" trigger, the wild zebra faced much greater danger from predators (lions) and had its trigger set much more on "flight" than "fight" than the horse, which is also one of the reasons they are so much harder to domesticate. Further, the fact they were lion food also motivated early humans to leave them alone. Thus, the difference between wild zebras and horses is revealed as the differences in a social network with three actors: myself, the zebra (or horse), and the predator (or none in the wild plains horse's case). Personally, I would stay far away and in front of any of these animals, and would largely treat them all as equivalent, thank you very much.

\section{C.5 Theory of Mind}

A hybrid POMDP like BayesAct can account for both theory-theory and simulation-theory type approaches, but obviates the need for any "mentalizing" or "mindreading," while still allowing for it, as I will now explain. First, note that any first-order logical database can be converted to a non-probabilistic MDP in which rules are implemented as 1-0 probabilities. Thus, a theory may be developed that can make logical predictions of the future based on a logical framework. Reasoning directly at the lifted level is also possible (Getoor and Taskar, 2007), however in both cases logical deductions based on this theory will be "fuzzy" in the probabilistic sense, and agents will always maintain some doubt that they may in fact be wrong. On the other hand, a POMDP can be easily uesed to run simulations into the future, a technique often used for reinforcement leraning using Monte-Carlo tree search (Silver and Veness, 2010). The key innovation in BayesAct is that the connotative state is used to "illuminate" a part of the denotative space, which can then simulate "rollouts" into the future within the illuminated

\footnotetext{
${ }^{12}$ This story is not as fanciful as it sounds. If I am in a mall and see a life-sized wooden statue of a man in a costume with a feather headdress, I will know it is a cigar store, even though the statue doesn't involve any cigars at all. As another example, consider the rod of Asclepius and note that it is equally improbable that a stick entwined with a snake came to symbolize the modern colleges of western medicine, and yet one would agree the two are inextricably linked by a common meaning.
} 
space (Asghar and Hoey, 2015). The illumination comes from the social-psychological model of $\mathrm{ACT}$, and so represents a logical framework (actor-behaviour-object), which can be extended to include other elements than people, behaviour and settings (Shank and Lulham, 2016). Thus, it is a theory-theory type of prediction: it predicts the abstract and general outcomes, which may be sufficient to make a decision. Otherwise simulation-theory kicks in to explore futures within the illuminated space. This is close to what Slors (2012) refers to as a "minimal theory of mind" theory in which "we have one system for computationally efficient but inflexible mindreading and another system for flexible but cognitively demanding mindreading" (Slors, 2012, p.20). BayesAct uses precise connotative predictions (efficient, but denotatively constrained) as the first, with precise denotative predictions as the second (slow, denotatively unconstrained). BayesAct is a "non-mentalizing social cognitive process" (Slors, 2012, p.16) that uses socially constrained beliefs and desires. While Dennett (1987)'s intentional stance means a system's behavior is understood as being issued by beliefs and desires, BayesAct adds to this "[...] issued by beliefs and desires within the limited set of futures given by the connotative system (cultural structure)."

Mindreading, or the ability to maintain a theory of mind about someone else, is commonly held to be a fundamental property of human intelligence, and underlies most discussions of both simulation- and theory-theories. That is, I predict what someone else will do by having a complete model of their mind inside my mind. Attempts to do this using a nested POMDP have generated impressive computational results, but have met with very limited success on real problems because of the intractability of the infinite regress so created (Doshi and Gmytrasiewicz, 2009). The phenomenological view counters this, however, by noting that much of theory of mind is not necessary in order to predict what others will do (Slors, 2012; Kiverstein, 2011). Kiverstein (2011, p.46) explicitly says that "[...] I am engaged with the world in the sense that my perception is of something that matters to me in such a way as to draw an action from me," and this drawing of an action as a "pre-objective mode of experience" or a "field of meanings or action possibilities." This would imply, in my reading, that the "da-sein" of Heidegger (1927), or "being-in-the world," is actually a sector of a partition of an agent's action space. That is, it is a region of an agent's action space that contiguously is mapped to from many different sensory inputs, all leading to the same action in the world. Kiverstein (2011, p47,n11) also point to the "ontological priority of reality as it is disclosed to us pre-objectively." and note that "objective thought is an abstraction from pre-objective modes of perception" Kiverstein (2011, p.47). Thus, it does seem the phenomenological views are focussed at the sensory input level of BayesAct, as I now explain.

A BayesAct model assumes, in the simplest case, that the "flashlight" being used by another agent is the same as its own. This does not mean I will have the same thoughts as my neighbour Chad in the same situation. It means that I believe I am using the same model of cultural structure as Chad in order to know where to look for options for behaviour in the situation. I expect Chad to behave as neighbours behave after a snowfall (we help each other shoveling snow), but as artists behave when he is working in his studio, as husbands behave on valentine's day, and as fathers behave when he is taking his daughter somewhere, etc. I do not need to have a model of what is in Chad's brain to do this. I simply need a model of the cultural structure and how it relates to the social structure and to the objective world. That means that I will make the same predictions about snow shoveling if Chad moves out and an unmarried childless American female engineer moves in, but my valentine's day predictions may be quite different. In the end, my engagement with the social world is much more about categorizing people within a cultural 
structure than it is about figuring out what they are thinking. As noted by (Shapira et al., 2012, p.233) "Using group-based categories implies that information on group belongingness is central, whereas individuating information is secondary and mutable. [...] the target of such categorization is equivalent to to other members of that category, and that those of his or her characteristics that are not inferable from category membership are secondary." BayesAct takes the identity of participants in a cultural structure to be the basis for action selection, and obviates the need for mentalizing by starting from a class of models, and an assignment of identities, that is already in agreement across participants. Thus, the outfielder (baseball player) who dynamically adjusts his position as he runs to catch a fly ball according to the angle of the ball in his line of sight (Gigerenzer and Gray, 2017), also dynamically adjusts his position as he generates expectations for the first baseman. As he does not apply Newton's equations of motion to the ball, he does not need to calculate what is going through the first baseman's head, because all that matters to him is the projection of any first-baseman onto his action space. So long as he maintains his action within a certain range, he will successfully deliver the ball to a competent first baseman. This is precisely what "first baseman" means to the outfielder in the context of a recently caught fly ball. It is this projection onto the action space, shared by all the players, which consitutes the cultural structure, or the "background of embodied practices" (Kiverstein, 2011, p.59) In any case, I leave the debate about theory-theory and simulation-theory aside for now, as I believe the two can be easily combined under a single umbrella. However, interested readers can consult a wealth of literature starting from (Kiverstein, 2011).

\section{C.6 Bias-Variance in Machine Learning}

Finally, there is a slight wrinkle in the bias variance story that requires some explanation. Recent results have shown that in the overparameterized regime ${ }^{13}$ the bias-variance effect is reversed, and lowering bias starts to decrease variance (Neal et al., 2018). This is because, in fact, variance arises for two reasons. First, variance due to sampling is what I have described above: if different datasets are gathered, then the results are different. This variance is reduced by gathering more data (or equivalently increasing bias relative to the dataset size). This type of variance continues to increase as bias is decreased beyond the overparametrization boundary defined by the VC dimension. The second type of variance is due to optimization, and arises because the process of fitting a model to a set of data is not deterministic for complex models, and may yield different results each time. This type of variance apparently starts to decrease in the overparameterized regime. The sum of both variances can be shown to decrease: the variance due to optimization plays a bigger role than the variance due to sampling once the model is overparameterized. These results are still being investigated, but I close by noting that it makes little difference for models of human intelligence, as these are highly unlikely to be able to be overparameterized given the complexity of the data encountered in a human life.

\footnotetext{
${ }^{13}$ Overparametrized models are more complex than required by the VC dimension (a measure of model capacity). Thus, a polynomial of degree two is overparameterized for data that lie in a straight line.
} 